\title{
Prevalence of Yersinia enterocolitica and Yersinia pseudotuberculosis in wild boars in the Basque Country, northern Spain
}

\author{
Maialen Arrausi-Subiza, Xeider Gerrikagoitia, Vega Alvarez, Jose Carlos Ibabe and Marta Barral
}

\begin{abstract}
Background: Yersiniosis is a zoonosis widely distributed in Europe and swine carry different serotypes of Yersinia enterocolitica and Y. pseudotuberculosis. The aim of this study was to determine the prevalence of Y. enterocolitica and $Y$. pseudotuberculosis in wild boars in northern Spain. The blood of wild boars $(n=505)$ was sampled between 2001 and 2012. Seroprevalence was determined in 490 serum samples with an indirect enzyme-linked immunosorbent assay. Seventy-two of the animals were also examined for the presence of $Y$. enterocolitica or Y. pseudotuberculosis in the tonsils with real-time polymerase chain reaction. All the tonsils were analysed twice, directly and after cold enrichment in phosphate-buffered saline supplemented with $1 \%$ mannitol and $0.15 \%$ bile salts.

Results: Antibodies directed against Y. enterocolitica and Y. pseudotuberculosis were detected in $52.5 \%$ of the animals. Yersinia enterocolitica was detected with real-time polymerase chain reaction in $33.3 \%$ of the wild boars and $Y$. pseudotuberculosis in $25 \%$. Significant differences were observed according to the sampling year, and the highest prevalence was during winter and spring. The highest antibody levels and $Y$. enterocolitica prevalence were observed in mountainous areas at altitudes higher than $600 \mathrm{~m}$, with very cold winters, and with the highest annual rainfall for each dominant climate. Areas with low and medium livestock populations were associated with the highest seroprevalence of Yersinia spp. in wild boars, whereas areas with high ovine populations had the highest prevalence of $Y$. enterocolitica.
\end{abstract}

Conclusions: This study shows that Y. enterocolitica and Y. pseudotuberculosis are highly prevalent among wild boars in the Basque country, with Y. enterocolitica most prevalent. The risk of infection among wild boars is influenced by the season and the area in which they live.

Keywords: Yersinia enterocolitica, Yersinia pseudotuberculosis, Wild boar, Epidemiology, PCR, ELISA

\section{Background}

Yersiniosis is the fourth most frequently reported foodborne zoonosis in humans in Europe, although the number of reported cases of Yersinia infection has continued to decrease since 2007 [1]. The genus Yersinia is composed of several species, but only Y. pestis, Y. pseudotuberculosis and some $Y$. enterocolitica strains are human pathogens [1].

Pigs are assumed to be the main reservoir of human pathogenic $Y$. enterocolitica, and serotypes isolated from

*Correspondence: mbarral@neiker.eus

Department of Animal Health, Basque Institute for Agricultural Research

and Development-NEIKER, Berreaga 1, 48160 Derio-Bizkaia, Spain pig samples, such as $4 / 0: 3$, are the same that cause human disease in Europe [1]. Yersinia pseudotuberculosis has also been frequently isolated from pigs and these animals might be a source of human 2/O:3 infections [2].

Wild animals constitute a very important factor in the epidemiology of Yersinia infection [3, 4], and wild boars (Sus scrofa) are considered an important reservoir of enteropathogenic Yersinia [5]. A great variety of serotypes, including those that cause human infections, have been isolated from wild boars in Europe $[3,5,6]$, although some $Y$. enterocolitica strains differ from those in domestic pigs [2]. 
More studies are required to understand the real role of wild boars in the epidemiology of yersiniosis. During the last two decades, the wild boar population has increased significantly in Europe [7], favouring their contact with livestock and the transmission of diseases [8]. Interest in wild boars as a meat source has also increased, thus increasing the risk of the transmission of food-borne diseases [9].

The prevalence of pathogenic Yersinia spp. in Spanish wild boars is unknown. Therefore, the aim of this study was to determine the prevalence of $Y$. enterocolitica and $Y$. pseudotuberculosis in wild boars in northern Spain.

\section{Methods}

\section{Study area}

The Basque country is located in northern Spain, limited by the Cantabrian coastline and distributed in eight regions, defined according to rainfall, temperature, altitude and the dominant vegetation $[10,11]$. Climatologically, the Atlantic slope (northern part) is moderate in terms of temperature, but very rainy, whereas the Mediterranean slope (southern part) is less rainy, with warmer summers and colder winters.

\section{Sample collection}

Wild boar samples were collected within the context of a wildlife health surveillance program in the Basque Country. In total, 505 wild boars were sampled between 2001 and 2012, during which time 490 serum samples were obtained, and in the last 3 years, 72 tonsils were also collected. Both serum and tonsil samples were obtained from only 57 animals. Most of the animals studied (90\%) had been shot by accredited hunters, and samples were taken in the field in collaboration with competent local authorities, and $8 \%$ were obtained from wildlife rehabilitation centres. The cause of death and the health status of these animals were not recorded. The remaining samples ( $2 \%$ ) were obtained from animals found dead or run over, and necropsies were performed in the laboratory. No significant lesions, except physical trauma, were observed in these animals. The samples were collected in individual containers, properly identified and stored at $-20{ }^{\circ} \mathrm{C}$ until analysis. The details of each animal, including its sex, age, and the date and geographic location of collection were recorded. The animals were classified into two groups according to age: young, including piglets $(<1$ year) and yearlings ( $1-2$ years); and adults ( $>2$ years). Details of the animals are given in Tables 1 and 2 .

\section{Real-time polymerase chain reaction}

The tonsil samples (1-5 g) were weighed and aseptically cut into small pieces. Approximately $150 \mathrm{mg}$ of each tonsil was disrupted and homogenised with 30 chrome-steel beads (1.3 mm) (Biospec Products, Bartlesville, OK, USA) and $750 \mu \mathrm{L}$ of TE buffer using the TissueLyser system (Qiagen, Hilden, Germany). DNA was extracted from $200 \mu \mathrm{L}$ of the supernatant for direct real-time polymerase chain reaction (rt-PCR) analysis. The rest of each tonsil sample was mixed with phosphate-buffered saline (PBS) supplemented with $1 \%$ mannitol (Fluka, Seelze, Germany) and $0.15 \%$ bile salts (Fluka, Seelze, Germany) (PBS-MSB), diluted 1:10 and homogenised in a stomacher (Lab-Blender 80, Cole-Parmer, Vernon Hills, IL, USA) until homogeneity. The mixture was incubated for 14 days at $4{ }^{\circ} \mathrm{C}$. DNA was extracted from $200 \mu \mathrm{L}$ of the supernatant and used as the template for rt-PCR.

DNA extraction was performed with the QIAamp ${ }^{\circledR}$ DNA Blood Mini Kit (Qiagen), according to the manufacturer's instructions, with minor modifications [12], and the DNA was measured with a NanoDrop ND-1000 spectrophotometer (Thermo Scientific, Inc.). DNA (150$200 \mathrm{ng}$ ) was used to detect Yersinia with the TaqMan rt-PCR assay in three independent reactions, using the Applied Biosystems 7500 Real-Time PCR System and The Express qPCR Supermix, universal kit (Invitrogen ${ }^{\mathrm{TM}}$ ), according to the supplier's recommendations. Yersinia enterocolitica was detected with the amplification of the ail gene [13], using a previously described procedure [12]. To detect all the $Y$. pseudotuberculosis serotypes, the $w z z$ and ail genes were amplified in two independent reactions $[12,14,15]$. Amplification of the ail gene detects all serotypes but O:11 and O:12, and amplification of the $w z z$ gene detects all serotypes but O:6 and O:7 [14, 15]. A sample was considered positive for $Y$. enterocolitica or $Y$. pseudotuberculosis when at least one positive result was obtained in the direct reaction or after enrichment in any of the three rt-PCRs used.

\section{Enzyme-linked immunosorbent assay}

The presence of antibodies directed against pathogenic Yersinia was determined with a commercial indirect enzyme-linked immunosorbent assay (ELISA) specific for swine (PIGTYPE ${ }^{\circledR}$ YOPSCREEN, Labor Diagnostic, Leipzig, Germany), according to the manufacturer's instructions. The optical density (OD) was measured in an ELISA Multiskan (Thermo Labsystem) spectrophotometer at $450 \mathrm{~nm}$. The ratio between the sample OD and the positive control OD (S/P ratio) was calculated. Samples with an $S / P$ ratio $\geq 0.3$ were considered positive.

\section{Bacteriology}

Selective cefsulodin-irgasan-novobiocin (CIN) agar (bioMérieux, Marcy l'Etoile, France) and CHROMagar ${ }^{\text {TM }}$ $Y$. enterocolitica (CHROMagar, Paris, France) agar were inoculated with $20 \mu \mathrm{L}$ of the rt-PCR-positive tonsil mixtures and incubated at $30{ }^{\circ} \mathrm{C}$ for $24-48 \mathrm{~h}$ to isolate the 
Table 1 Seroprevalence of pathogenic Yersinia spp. detected in wild boars according to the variables studied

\begin{tabular}{l} 
Variables \\
\hline Age \\
Young \\
Adult \\
Sex \\
Females \\
Males \\
Sampling year \\
2001 \\
2002 \\
2003 \\
2004 \\
2005 \\
2006 \\
2010 \\
2011 \\
2012 \\
Season \\
Winter \\
Spring \\
Summer \\
Autumn
\end{tabular}

Natural regions

1

2290

1

4

6

Slope

Atlantic

Mediterranean

Porcine census

Low (10-140)

Middle (167-426)

High (580-7332)

Caprine census

Low (66-655)

Middle (909-1056)

High (1136-2810)

Ovine census

\begin{tabular}{lll} 
Low $(1881-6698)$ & 102 & $60(58.8)$ \\
Middle $(8035-15,033)$ & 138 & $91(65.9)$ \\
High $(15,417-32,802)$ & 222 & $94(42.3)$ \\
Bovine census & & \\
Low $(276-4277)$ & 132 & $77(58.3)$ \\
Middle $(4602-6768)$ & 172 & $103(59.9)$ \\
High $(6781-19,109)$ & 158 & $65(41.1)$ \\
\hline
\end{tabular}

$N$ number of samples analyzed, ELISA number and percentage of ELISA positive samples
Table 2 Prevalence of pathogenic Yersinia detected with rt-PCR in wild boars according to the variables studied

\begin{tabular}{|c|c|c|c|c|}
\hline Variables & $\mathrm{N}$ & YE and YP (\%) & YE (\%) & YP (\%) \\
\hline \multicolumn{5}{|l|}{ Age } \\
\hline Young & 25 & $18(72)$ & $12(48)$ & $9(36)$ \\
\hline Adult & 20 & $10(50)$ & $8(40)$ & $3(15)$ \\
\hline \multicolumn{5}{|l|}{ Sex } \\
\hline Females & 30 & $19(63.3)$ & $12(40)$ & $11(36.7)$ \\
\hline Males & 19 & $12(63.2)$ & $9(47.4)$ & $4(21.1)$ \\
\hline \multicolumn{5}{|c|}{ Sampling year } \\
\hline 2010 & 23 & $18(78.3)$ & $13(56.5)$ & $9(39.1)$ \\
\hline 2011 & 32 & $7(21.9)$ & $7(21.9)$ & 0 \\
\hline 2012 & 17 & $12(70.6)$ & $4(23.5)$ & $9(52.9)$ \\
\hline \multicolumn{5}{|l|}{ Season } \\
\hline Winter & 8 & $5(62.5)$ & $5(62.5)$ & $1(12.5)$ \\
\hline Spring & 9 & $7(77.8)$ & $2(22.2)$ & $6(66.7)$ \\
\hline Summer & 10 & $5(50)$ & $3(30)$ & $2(20)$ \\
\hline Autumn & 45 & $20(44.4)$ & $14(31.1)$ & $9(20)$ \\
\hline \multicolumn{5}{|c|}{ Natural regions } \\
\hline 1 & 58 & $26(44.8)$ & $15(25.9)$ & $15(25.9)$ \\
\hline 2 & 6 & $5(83.3)$ & $5(83.3)$ & 0 \\
\hline \multicolumn{5}{|l|}{ Slope } \\
\hline Atlantic & 72 & $37(51.4)$ & $24(33.3)$ & $18(25)$ \\
\hline \multicolumn{5}{|c|}{ Porcine census ${ }^{\mathrm{a}}$} \\
\hline Low & 26 & $12(46.1)$ & $6(23.1)$ & $8(30.8)$ \\
\hline Middle & 24 & $11(45.8)$ & $6(25)$ & $6(25)$ \\
\hline High & 22 & $14(63.6)$ & $12(54.6)$ & $4(18.2)$ \\
\hline \multicolumn{5}{|c|}{ Caprine census $^{b}$} \\
\hline Low & 3 & 0 & 0 & 0 \\
\hline Middle & 35 & $19(54.3)$ & $10(28.6)$ & $12(34.3)$ \\
\hline High & 34 & $18(52.9)$ & $14(41.2)$ & $6(17.7)$ \\
\hline \multicolumn{5}{|c|}{ Ovine census ${ }^{c}$} \\
\hline Low & 20 & $7(35)$ & $3(15)$ & $4(20)$ \\
\hline Middle & 28 & $12(42.9)$ & $6(21.4)$ & $8(28.6)$ \\
\hline High & 24 & $18(75)$ & $15(62.5)$ & $6(25)$ \\
\hline \multicolumn{5}{|c|}{ Bovine census ${ }^{d}$} \\
\hline Low & 26 & $13(50)$ & $6(23.1)$ & $9(34.6)$ \\
\hline Middle & 17 & $7(41.2)$ & $4(23.5)$ & $3(17.7)$ \\
\hline High & 29 & $17(58.6)$ & $14(48.3)$ & $6(20.7)$ \\
\hline
\end{tabular}

$N$ number of samples analyzed, $Y E$ and $Y P$ number and percentage of $Y$. enterocolitica and $Y$. pseudotuberculosis positive samples, YE number and percentage of $Y$. enterocolitica positive samples, YP number and percentage of $Y$. pseudotuberculosis positive samples

a Porcine census: low (10-140), middle (167-426), high (580-7332)

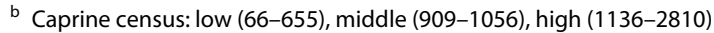

c Ovine census: low (1881-6698), middle (8035-15,033), high $(15,417-32,802)$

d Bovine census: low (276-4277), middle (4602-6768), high (6781-19,109) 
Yersinia strains. Red CIN agar "bull's-eye" colonies surrounded with a transparent area of $1 \mathrm{~mm}$ and mauve CHROMagar $^{\mathrm{TM}}$ colonies were selected. The selected colonies were homogenised in $500 \mu \mathrm{L}$ of PBS, and $50 \mu \mathrm{L}$ of this mixture was incubated for $10 \mathrm{~min}$ at $100{ }^{\circ} \mathrm{C}$ in a water bath and then for $10 \mathrm{~min}$ on ice. The mixture was then centrifuged for $10 \mathrm{~min}$ at $15,600 \times g$ and $5 \mu \mathrm{L}$ of the supernatant was used for $Y$. enterocolitica and $Y$. pseudotuberculosis identification with rt-PCR, with the procedures described above. The colonies were also streaked directly onto triple sugar iron agar (Oxoid Ltd, Basingstoke, England) and onto blood agar (bioMérieux) and identified with the VITEK system (bioMérieux), using a previously reported protocol [12].

The Yersinia strains were serotyped with slide agglutination using commercial $Y$. enterocolitica O:1, O:2, O:3, O:5, O:8 and O:9 antisera (Denka Seiken, Coventry, UK), Y. enterocolitica O:27 antiserum (SIFIN, Berlin, Germany) and Y. pseudotuberculosis O:1-O:6 antisera (Denka Seiken). Yersinia pseudotuberculosis was also serotyped with O-genotyping, using a conventional multiplex PCR, according to Bogdanovich et al. [16].

\section{Data analysis}

All statistical analyses were performed in the SAS 9.3 software. The official 2009 livestock census data were obtained from the Basque Statistics Institute (http://www.eustat.es) for each region and the PROC RANK Statement was used to classify each region as containing high, medium, or low numbers of each species. The relationships between Yersinia prevalence and the different independent variables studied (sex, age, sampling year, season, natural region, slope and livestock numbers) were examined statistically using the $\chi^{2}$ or Fisher's test. The simple kappa coefficient of agreement was used to determine the degree of agreement between the ELISA and PCR results when applied to the same animal. A $t$ test was used to compare the ELISA S/P ratios between the PCR-positive and -negative animals. Differences were considered significant at $P<0.05$.

\section{Results}

Antibodies directed against pathogenic Yersinia were detected in $52.5 \%(257 / 490)$ of the wild boars. The mean $\mathrm{S} / \mathrm{P}$ ratio was 0.66 (95\% confidence interval [CI] 0.630.70 ) for the ELISA-positive samples and 0.061 (95\% CI 0.05-0.07) for the ELISA-negative samples (Fig. 1).

Yersinia infection was detected with rt-PCR in $51.4 \%$ (37/72) of wild boars. Yersinia enterocolitica was present in $33.3 \%(24 / 72)$ and Y. pseudotuberculosis in $25.0 \%(18 / 72)$ of the animals. Mixed infections of $Y$. enterocolitica and $Y$. pseudotuberculosis were identified in five individuals. Ten of the $18 Y$. pseudotuberculosis-positive samples were detected with the amplification of both the ail and $w z z$

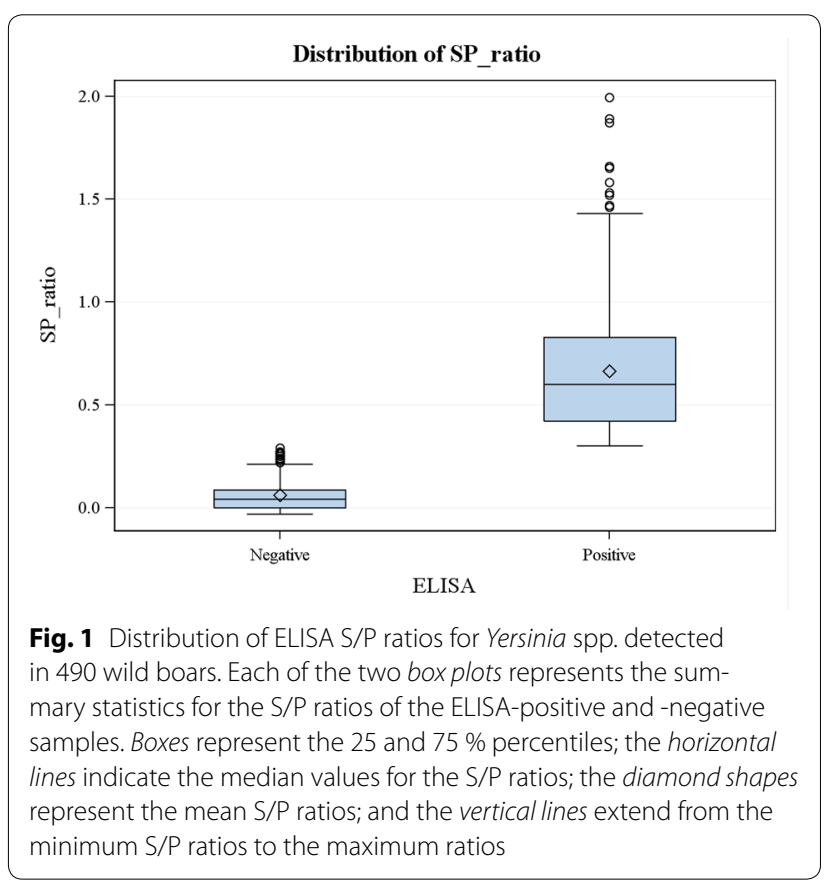

genes, four with the amplification of only ail, and the other four with the amplification of only the $w z z$ gene.

Of the 37 rt-PCR-positive samples, 23 were only positive after enrichment and nine were only positive on direct rt-PCR. Eight samples were positive on both direct rt-PCR and after enrichment, but lower cycle threshold $(\mathrm{Ct})$ values were obtained after enrichment (see Additional file 1).

Seroprevalence was higher in the adult animals than in the young animals $(P<0.0001$; Table 1$)$, but no significant differences were observed according to age with PCR $(P=0.2157$; Table 2$)$.

Significant differences were observed according to the sampling year. The highest seroprevalence was detected in 2002 and in 2005-2006, although in 2002, only 10 samples were analysed $(P<0.0001$; Table 1$)$. The prevalence of $Y$. enterocolitica was highest in $2010(P=0.0213)$ and that of $Y$. pseudotuberculosis was highest in 2012 $(P<0.0001$; Table 2).

The overall seroprevalence was highest in winter and spring $(P<0.0001$; Table 1$)$. The prevalence of $Y$. pseudotuberculosis was highest in spring $(P=0.0305)$, but no significant difference was observed in the prevalence of $Y$. enterocolitica between seasons $(P=0.3180$; Table 2$)$.

Statistically significant differences were observed in the seroprevalence of Yersinia spp. according to the slope and region of habitation $(P<0.0001$; Table 1$)$. These differences were also significant for $Y$. enterocolitica and regions $(P=0.0096$; Table 2$)$. The geographic distribution of the positive samples is illustrated in Fig. 2. 


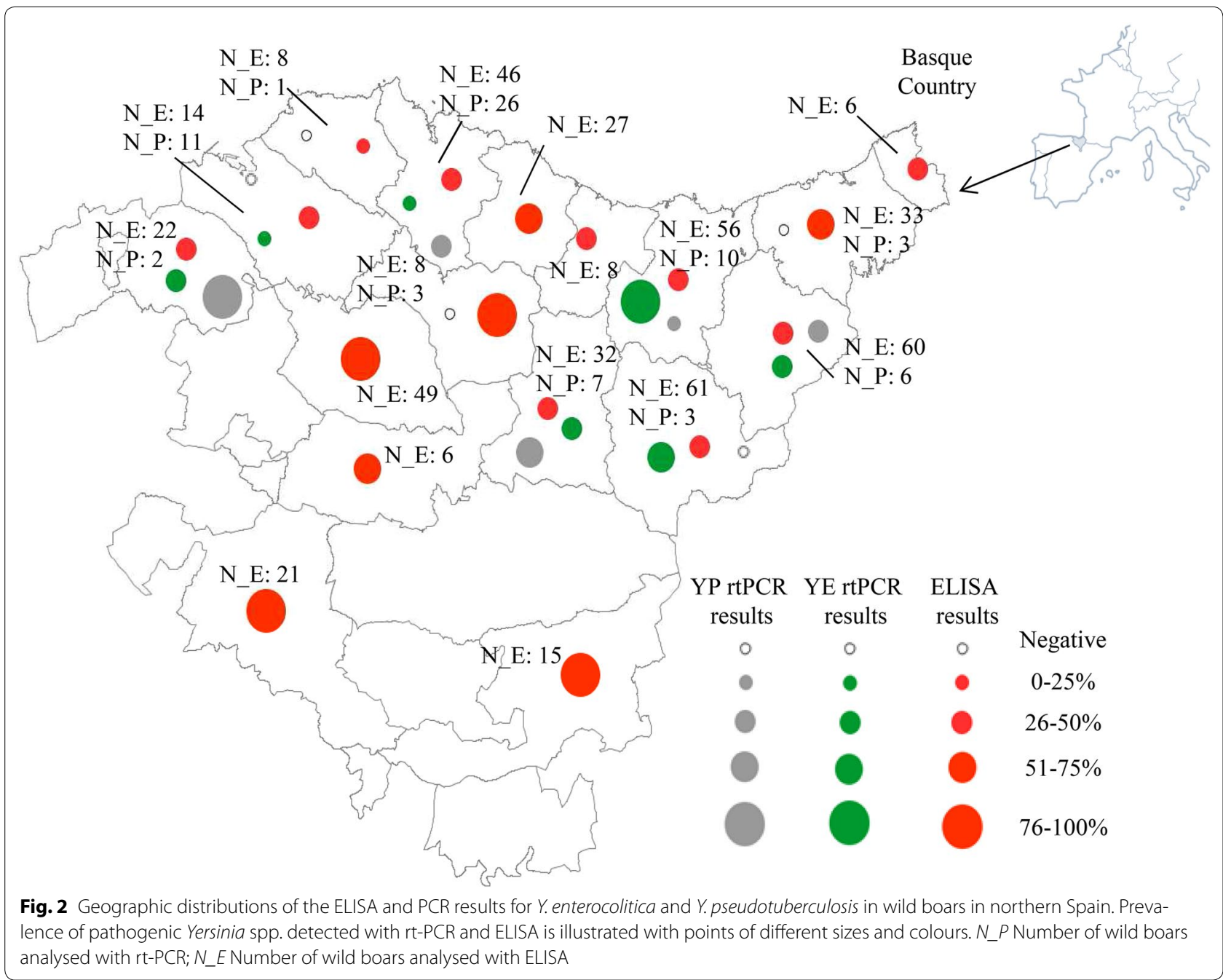

Higher seroprevalence was observed in areas with small livestock populations (caprine, $P<0.0001$ ) or medium livestock populations (bovine, $P=0.0011$; ovine, $P<0.0001$ ) (Table 1), whereas $Y$. enterocolitica prevalence was highest in areas with large ovine populations $(P=0.0012$; Table 2).

Two isolates of $Y$. pseudotuberculosis and two of $Y$. enterocolitica were collected from four different wild boars. The $Y$. pseudotuberculosis isolates were obtained on CIN agar, one with direct plating and the other after enrichment. Both $Y$. enterocolitica isolates were obtained after enrichment, one on CIN agar and the other on CHROMagar ${ }^{\mathrm{TM}}$. The identities of $Y$. enterocolitica and Y. pseudotuberculosis were confirmed for each isolate with rt-PCR amplification of the ail gene. No agglutination was detected when the
Y. pseudotuberculosis isolates were serotyped with the antisera used, but both isolates were identified as serotype O:1c with multiplex O-gene amplification. It was not possible to serotype the $Y$. enterocolitica isolates because of contamination.

Of the 57 wild boars analysed with rt-PCR and ELISA, 13 were positive and 19 were negative with both techniques, seven animals were positive only according to ELISA, and 18 animals were positive only according to rtPCR ( $\kappa$ index $=0.1452$ ). No differences were observed in the ELISA S/P ratios when the Y. enterocolitica-rt-PCRpositive and -negative animals were compared. However, the $Y$. pseudotuberculosis-positive animals had higher S/P ratios (mean 0.53; $95 \%$ CI 0.21-0.86) than the Y. pseudotuberculosis-negative animals (mean 0.23; $95 \%$ CI $0.12-$ $0.35 ; P=0.0249$ ). 


\section{Discussion}

This study demonstrates that $Y$. enterocolitica and $Y$. pseudotuberculosis infections are widespread among the wild boars in northern Spain. The seroprevalence was high (52.5\%), although slightly lower than those detected in wild boars in Germany and Switzerland (62.6 and $65.0 \%$, respectively) $[5,17]$. The prevalence of $Y$. enterocolitica and $Y$. pseudotuberculosis can also be considered high ( 33.5 and $25 \%$, respectively) because their observed prevalence in wild boars in Europe ranges from 4.35 to $35 \%$ for Y. enterocolitica and is around $20 \%$ for Y. pseudotuberculosis [5, 18, 19]. Yersinia enterocolitica was more prevalent than Y. pseudotuberculosis, as is usually found in wild boars and pigs [5, 20]. Mixed infections were detected in a proportion of the animals, as previously described [5], but the prevalence of Y. pseudotuberculosis (25\%) was higher than expected in wild boars or organically produced pigs, probably because they are in frequent contact with other infected wild species and livestock in extensive grazing systems [4, 21]. The use of two different rt-PCR methods and the higher detection rates recorded when an enrichment step was included before rt-PCR, could also have improved the detection rate for Y. pseudotuberculosis [12].

The highest seroprevalence was detected in spring and winter, which is attributable to the highest $Y$. pseudotuberculosis prevalence recorded in spring and the (not significantly) highest $Y$. enterocolitica prevalence recorded in winter. To the best of our knowledge, the seasonality of $Y$. enterocolitica and $Y$. pseudotuberculosis infections has not been reported previously in wild boars. However, in other wildlife species, the disease is usually detected in the coldest months of the year [22] or from November to May, which is related to the birth of newborns [23].

The highest seroprevalence and presence of $Y$. enterocolitica were associated with mountainous areas at altitudes higher than $600 \mathrm{~m}$, very cold winters, and the highest annual rainfall for each dominant climate. A similar trend was observed in pigs slaughtered in China, in which the incidence of $Y$. enterocolitica was higher in cold areas than in warm areas [24].

The highest prevalence of $Y$. enterocolitica was detected in areas with a high ovine presence. Sheep have been described as a reservoir of pathogenic $Y$. enterocolitica and $Y$. pseudotuberculosis [25, 26], but little is known about the infection of sheep in Spain with pathogenic Yersinia or their relationship with the Yersinia species found in wild boars, although Yersinia is reported to cause sporadic abortion in sheep in the area studied [27]. In contrast, the highest Yersinia seroprevalence was associated with medium or low numbers of other livestock, suggesting that other wildlife species also contribute to the epidemiology of Yersinia infection among wild boars.
However, more studies are required to determine the real impact of pathogenic Yersinia on livestock in this area.

The rates of isolation were low, despite the use of two different culture media, including CHROMagar ${ }^{\mathrm{TM}}$, which is recommended for the isolation of $Y$. enterocolitica [28]. Y. enterocolitica pathogenicity therefore remains unknown, because the ail gene is an insufficient marker of virulence, and is also present in some $Y$. enterocolitica biotype 1A strains [29]. The only two Y. pseudotuberculosis strains isolated were identified as serotype O:1c. Little is known about the infection of animals or humans by serotype O:1c because the majority of studies have not included this subserotype. However, Y. pseudotuberculosis serotype O:1 has been described as one of the most commonly found serotypes infecting wild boars, pigs and humans in Europe [2, 30,31]. This fact highlights the need for the better characterisation of its pathogenicity.

More efforts are required to isolate and characterise the Yersinia strains from infected wild boars in the Basque country to determine their pathogenicity and any potential risk they pose to humans and domestic species.

\section{Conclusions}

This study demonstrates that $Y$. enterocolitica and $Y$. pseudotuberculosis are highly prevalent among wild boars in the Basque Country, with $Y$. enterocolitica the most frequently found species. The risk of infection among wild boars is influenced by the season and the area in which the animals live.

\section{Additional file}

Additional file 1. ELISA and rt-PCR results for 72 wild boars in which tonsils were available. Detailed information on the rt-PCR results and cycle threshold values for samples obtained from 72 wild boars in which tonsils were available. The ELISA results and S/P ratios obtained from the animals are also included.

\section{Authors' contributions}

MA, XG, VA and JCI performed the laboratory analyses. MA and MB performed the statistical analyses and wrote the manuscript. XG and VA participated in writing the manuscript. MA and MB conceived and designed the experiments. MB coordinated and supervised the study. All the authors participated in the interpretation of the results. All authors read and approved the final manuscript.

\section{Acknowledgements}

The study was supported by the Spanish National Institute for Agricultural and Food Research and Technology (INIA, RTA2010-00022), the Department of Agriculture and Fisheries (Basque Government) and European Regional Development Fund (ERDF). Maialen Arrausi-Subiza is the recipient of a predoctoral fellowship from "Fundación Candido de Iturriaga y María de Dañobeitia". We would like to thank Zuriñe Pérez and Dr Raquel Atxaerandio for their technical support, Diputaciones Forales and hunters for their collection of samples and Dr Janine Miller for English editing of the manuscript.

\section{Competing interests}

The authors declare that they have no competing interests. 
Received: 28 May 2015 Accepted: 3 January 2016

Published online: 20 January 2016

\section{References}

1. EFSA (European Food Safety Authority), ECDC (European Centre for Disease Prevention and Control). The European union summary report on trends and sources of zoonoses, zoonotic agents and food-borne outbreaks in 2013. EFSA J. 2015;13:3991. doi:10.2903/j.efsa.2015.3991.

2. Fredriksson-Ahomaa M, Wacheck S, Bonke R, Stephan R. Different enteropathogenic Yersinia strains found in wild boars and domestic pigs. Foodborne Pathog Dis. 2011;8:733-7.

3. Nikolova S, Tzvetkov Y, Najdenski H, Vesselinova A. Isolation of pathogenic Yersiniae from wild animals in Bulgaria. J Vet Med B Infect Dis Vet Public Health. 2001;48:203-9.

4. Fukushima H, Gomyoda M. Intestinal carriage of Yersinia pseudotuberculosis by wild birds and mammals in Japan. Appl Environ Microbiol. 1991:57:1152-5.

5. Fredriksson-Ahomaa M, Wacheck S, Koenig M, Stolle A, Stephan R. Prevalence of pathogenic Yersinia enterocolitica and Yersinia pseudotuberculosis in wild boars in Switzerland. Int J Food Microbiol. 2009;135:199-202.

6. Magistrali CF, Cucco L, Manuali E, Sebastiani C, Farneti S, Ercoli L, Pezzotti G. Atypical Yersinia pseudotuberculosis serotype 0:3 isolated from hunted wild boars in Italy. Vet Microbiol. 2014;171:227-31.

7. Acevedo P, Escudero MA, Muñoz R, Gortazar C. Factors affecting wild boar abundance across an environmental gradient in Spain. Acta Theriol. 2006;51:327-36

8. Köppel C, Knopf L, Ryser MP, Miserez R, Thür B, Stärk KDC. Serosurveillance for selected infectious disease agents in wild boars (Sus scrofa) and outdoor pigs in Switzerland. Eur J Wildl Res. 2006;53:212-20.

9. Sales J, Kotrba J. Meat from wild boar (Sus scrofa L.): a review. Meat Sci. 2013;94:187-201.

10. Barandika JF, Berriatua E, Barral M, Juste RA, Anda P, Garcia-Perez AL. Risk factors associated with ixodid tick species distributions in the Basque region in Spain. Med Vet Entomol. 2006;20:177-88.

11. Gobierno Vasco. Vegetación de la comunidad autónoma del País Vasco. Vitoria: Servicio Central de Publicaciones del Gobierno Vasco; 1989.

12. Arrausi-Subiza M, Ibabe JC, Atxaerandio R, Juste RA, Barral M. Evaluation of different enrichment methods for pathogenic Yersinia species detection by real time PCR. BMC Vet Res. 2014;10:192.

13. Lambertz ST, Nilsson C, Hallanvuo S, Lindblad M. Real-time PCR method for detection of pathogenic Yersinia enterocolitica in food. Appl Environ Microbiol. 2008;74:6060-7.

14. Lambertz ST, Nilsson C, Hallanvuo S. TaqMan-based real-time PCR method for detection of Yersinia pseudotuberculosis in food. Appl Environ Microbiol. 2008;74:6465-9.

15. Matero P, Pasanen T, Laukkanen R, Tissari P, Tarkka E, Vaara M, Skurnik M. Real Time multiplex PCR assay for detection of Yersinia pestis and Yersinia pseudotuberculosis. APMIS. 2009;117:34-44

16. Bogdanovich T, Carniel E, Fukushima H, Skurnik M. Use of O-antigen gene cluster-specific PCRs for the identification and O-genotyping of Yersinia pseudotuberculosis and Yersinia pestis. J Clin Microbiol. 2003:41:5103-12.

17. Al Dahouk S, Nockler K, Tomaso H, Splettstoesser WD, Jungersen G, Riber $U$, et al. Seroprevalence of brucellosis, tularemia, and yersiniosis in wild boars (Sus scrofa) from north-eastern Germany. J Vet Med B Infect Dis Vet Public Health. 2005:52:444-55.

18. Bancerz-Kisiel A, Szczerba-Turek A, Platt-Samoraj A, Socha P, Szweda W. Application of multiplex PCR for the evaluation of the occurrence of Ail,
Ysta, and Ystb genes in Yersinia enterocolitica strains isolated from wild boars (Sus scrofa). Bull Vet Inst Pulawy. 2009;53:351-5.

19. Sannö A, Aspan A, Hestvik G, Jacobson M. Presence of Salmonella spp. Yersinia enterocolitica, Yersinia pseudotuberculosis and Escherichia coli O157:H7 in wild boars. Epidemiol Infect. 2014;142:2542-7.

20. Ortiz Martínez P, Fredriksson-Ahomaa M, Pallotti A, Rosmini R, Houf K, Korkeala H. Variation in the prevalence of enteropathogenic Yersinia in slaughter pigs from Belgium, Italy, and Spain. Foodborne Pathog Dis. 2011:8:445-50.

21. Laukkanen R, Ortiz Martínez P, Siekkinen KM, Ranta J, Maijala R, Korkeala H. Transmission of Yersinia pseudotuberculosis in the pork production chain from farm to slaughterhouse. Appl Environ Microbiol. 2008:74:5444-50.

22. Mair NS. Yersiniosis in wildlife and its public health implications. J Wildl Dis. 1973;9:64-71

23. Fukushima H, Gomyoda M, Kaneko S. Mice and moles inhabiting mountainous areas of Shimane Peninsula as sources of infection with Yersinia pseudotuberculosis. J Clin Microbiol. 1990;28:2448-55.

24. Liang J, Wang X, Xiao Y, Cui Z, Xia S, Hao Q, et al. Prevalence of Yersinia enterocolitica in pigs slaughtered in Chinese abattoirs. Appl Environ Microbiol. 2012;78:2949-56.

25. Nikolaou K, Hensel A, Bartling C, Tomaso H, Arnold T, Rosler U, et al. Prevalence of anti-Yersinia outer protein antibodies in goats in lower saxony. J Vet Med B Infect Dis Vet Public Health. 2005:52:17-24.

26. Slee KJ, Skilbeck NW. Epidemiology of Yersinia pseudotuberculosis and Y. enterocolitica infections in sheep in Australia. J Clin Microbiol. 1992:30:712-5.

27. Oporto B, Barandika JF, Hurtado A, Aduriz G, Moreno B, Garcia-Perez AL. Incidence of ovine abortion by Coxiella burnetii in northern Spain. Ann N Y Acad Sci. 2006;1078:498-501

28. Renaud N, Lecci L, Courcol RJ, Simonet M, Gaillot O. CHROMagar Yersinia, a new chromogenic agar for screening of potentially pathogenic Yersinia enterocolitica isolates in stools. J Clin Microbiol. 2013:51:1184-7.

29. Sihvonen LM, Hallanvuo S, Haukka K, Skurnik M, Siitonen A. The ail gene is present in some Yersinia enterocolitica biotype 1A strains. Foodborne Pathog Dis. 2011;8:455-7.

30. Wacheck S, Fredriksson-Ahomaa M, König M, Stolle A, Stephan R. Wild boars as an important reservoir for foodborne pathogens. Foodborne Pathog Dis. 2010;7:307-12

31. Rimhanen-Finne R, Niskanen T, Hallanvuo S, Makary P, Haukka K, Pajunen $S$, et al. Yersinia pseudotuberculosis causing a large outbreak associated with carrots in Finland, 2006. Epidemiol Infect. 2009;137:342-7.

\section{Submit your next manuscript to BioMed Central and we will help you at every step:}

- We accept pre-submission inquiries

- Our selector tool helps you to find the most relevant journal

- We provide round the clock customer support

- Convenient online submission

- Thorough peer review

- Inclusion in PubMed and all major indexing services

- Maximum visibility for your research

Submit your manuscript at www.biomedcentral.com/submit

( Biomed Central 